\title{
Presidential Lecture*
}

\section{Physicians and Epidemiology}

\author{
Noboru KIMURA**, MD
}

There are many approaches from various aspects to investigate the ecology of chronic diseases. One of them is the epidemiological approach, which is a very effective way to find etiology and preventive measures.

The epidemiological approach demands high reliability in its study. The vital statistics are also one of those which we can not evaluate highly from confidence limit. But the use of vital [statistics has played important role in cardiovascular epidemiology, by indicating where the main problems of ischaemic heart disease and stroke lie and by suggesting hypotheses for further investigation.

WHO, in which I had been a member of an expert adviser committee, published "International Classification of Diseases" in order to compare statistics from many countries. It has been revised with the progress in medicine every eight years. Old categories of disease tend to be remained in a new edition because of avoiding discontinuation in the statistics over long period. It took, for example, more than 15 years to categoralize so-called coronary atherosclerosis, coronary occulusion, and coronary insufficiency into ischaemic heart disease. Another pitfall is variance of physicians' ability even if the classification and the criteria of diseases are standardized.
We could not guarantee, however, that all physicians in the world are competent to give precise diagnos!s. It is therefore, not, meaningful to compare vital statistics in detail as they are.

In $1954 \mathrm{I}$ was invited to a symposium on "EPIDEMIOLOGY OF ATHEROSCLEROSIS" at the Second World Congress of Cardiology in Washinton DC. For this symposium, we examined all autopsied hearts preserved at the department of pathology in Kyushu University Medical School. Approximately 1000 postmortem hearts among them were kept in good shape, including coronary arteries.

From the WHO vital statistics for 1949, US white men aged 50's died from ischaemic heart disease ten times more than Japanese. Our postmortem study mentioned above showed less than $10 \%$ of prevalence rate of severe coronary atherosclerosis for male in their fifties. The prevalence rate of the same coronary atherosclerosis obtained from Mayo Clinic study, the method of which we applied to our postmortem study, exceeded $70 \%$.

These findings are identical with the figures in the vital statistics. This suggests that the ability in diagnosis of ischaemic heart disease is not much different between USA and Japan. What makes this distinct difference in the death of ischaemic heart

\footnotetext{
* Presented at the 75th Annual Meeting of the Japanese Society of Internal Medicine, May 12, 1978, in Fukuoka, Japan.

** President of the Japanese Society of Internal Medicine (1978) Vice President of Saga Medical School, Saga
} 
disease between these two countries?

At the same symposium, Professor Bjork from Sweden reported the corelation between the death from ischaemic heart disease and the low-fat intake by linking the following evidences. In the northern European countries the death rate of ischaemic heart disease declined sharply during the Second World War. Prior to this trend, the fat consumption also dropped in these countries due to the food requisition by the German Force.

For the next step, we investigated the autopsies of Japanese in Hawaii. Twentyfive percent of the prevalence rate of severe coronary atherosclerosis in the fifties among them was three times as much as it of Japanese in Kyushu.

Thus it is indicated that different modes of life influence prevalence of ischaemic heart disease even among the same race. We also suggested that fat rich diet was responsible for higher prevalence rate of severe coronary atherosclerosis of Japanese in Hawaii than those in Kyushu. Those epidemiological studies provided great impact on a research committee of the International Society of Cardiology and an expert committee of WHO to project population surveys in several countries with the same method under international cooperation.

The ideal population survey expects all residents in a selected area, having high degree of homogeneity in mode of life, to be examined. Consequently an urban area, high mobility in population and diversity in mode of life, is not considered a suitable area.

This internationally cooperated population survey, focusing on differences in life style especially diet, demanded the standardization of survey methods in order to compare data from several countries.

Fall in 1956, several investigators, directors of Seven Countries Study in their own countries later, met and performed a trial survey for this purpose at rural areas in southern Italy and Grete Island in
Greece.

Not long after returning to Japan from this trial survey, I left Kyushu University to found the Third Department of Internal Medicine, Kurume University Medical School, January 1958. In March, I initiated a longterm prospective population survey at a typical farming village in Tanushimaru, close to Kurume City. It took us three months to have done complete examinations of 639 subjects on the list, who were all the men aged 40 to 64 born and living in the prescribed area. It is quite important to have covered all the subjects. Now you assume that someone measures a half of all subjects for their blood pressures and reported $50 \%$ of the residents in this population as hypertensive. The percentage for high blood pressure, however, could not be $50 \%$ but $25 \%$ if the unexamined half are normotonsive !

Reasonably this example shows danger to believe figures obtained as above blindly. In fact, nevertheless, the frequent incidence of stroke in Japan has been discussed in connection with high incidence of hypertension without taking confidence limit to consideration. The converage of all subjects is essential from this reason in addition to standarization of survey methods.

Our survey in Tanushimaru, a typical farming village in Japan, became the first one named as Seven Countries Study afterward.

In 1960 we chose a fishing village in Ushibuka in Amakusa island for another survey to Seven Countries Study. This area differed from Tanushimaru in its dietary habit. We found following interesting points by analysing daily diet of subjects. The amounts of protein and fat intake in the farming village were a half of those in the fishing village while no great difference seen in total calories between these two areas.

As the result of 5,10 and 15 year follow-up studies, the difference in diet between two characteristic areas provide a clue to the question why in Japan the 
incidence of myocardial infarction is extremely low against the high incidence of stroke (Fig. 1, 2, 3).

The death rate from stroke for the fifties and sixties in Kyushu is twenty times higher for our population with high blood pressure than for those with normal blood pressure. It is, however, no more than 20 in 1,000 men with hypertension dies from stroke annually. This means majority of the men, having the same degree of high blood pressure, are not susceptible of stroke.

Dietary factor was raised as a key to solve this question in our study. As for stroke, the higher the percentage of protein and unsaturated fatty acid in diet, the lower the incidence rate of stroke in (Fig. 2, 3).

In other words mulnutrition due to the low intake of protein and unsaturated fatty acid gives a reason to stroke as keeping the top rank in cause of death in Japan. We believe our findings opened a way to the prevention of ischaemic heart disease and stroke as well as hypertension.

There is no need to say tremendous number of physicians played great role in obtaining valuable results. Unfortunately this kind of hard work does not seem to be fully appreciated in Japan.

Japanese Society of Internal Medicine, though considerably long time ago, did not

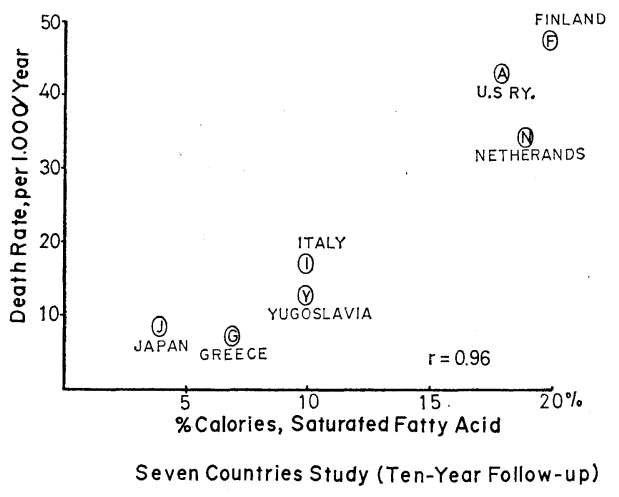

Fig. 1. Saturated Fatty Acid vs Ischemic Heart Disease

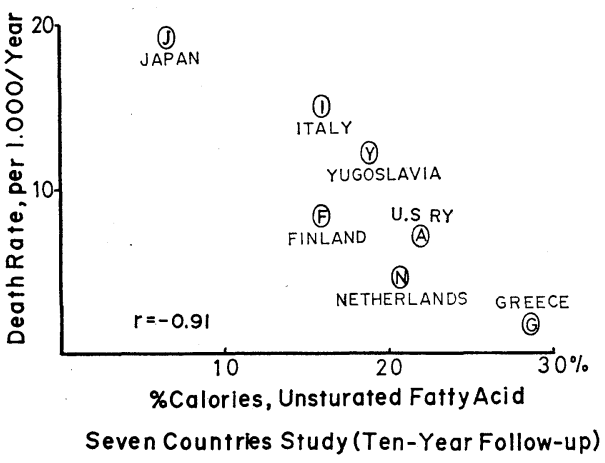

Fig. 2. Unsaturated Fatty Acid vs Stroke
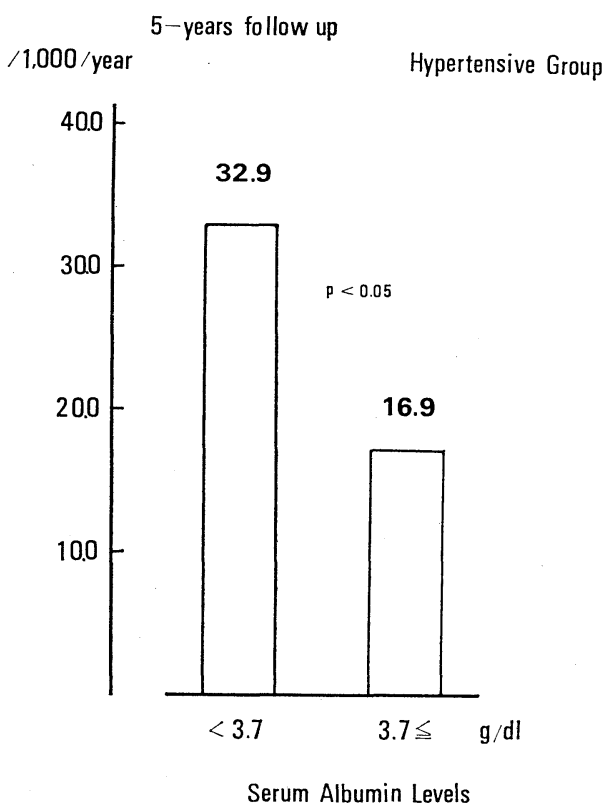

Fig. 3. Serum Albumin vs Stroke

accept a report on our population survey to its congress. I thought about various reasons for this rejection. One of them would be due to the characteristics of population survey. It is the "mad" field work directly dealing with human-beings, which consumes enormous energy and long length of time even entire life time of an investigator, in contrast with the work at laboratory under conditioned circumstances. If so, I believe we should ask ourselves once again "What is the physician?" "What is Internal Medicine?" 